\title{
Prevalência de burnout em médicos residentes de Medicina Geral e Familiar em Portugal
}

\author{
Burnout prevalence among Portuguese General and Family Medicine residents \\ Prevalencia del burnout en los médicos residentes de Medicina Familiar en Portugal
}

Sara Cristina Robalo dos Santos ${ }^{a}$, Ana Isabel Francisco Viegas ${ }^{b}$, Catarina Isabel de Magalhães Oliveira Morgadoa , Carla Sofia Varela Ramos ${ }^{a}$, Christina Nunes Delgado Soares ${ }^{c}$, Helena Mafalda da Conceição João Roxo d, Mafalda Cleto da Silva Santosa, Sara Nunes Pires Nabais ${ }^{b}$

\section{Resumo}

Objetivo: Determinar a prevalência de burnout nos residentes de Medicina Geral e Familiar (MGF) em Portugal e analisar variáveis que possam influenciar os níveis de burnout nas suas três dimensões (Exaustão Emocional EE; Despersonalização - DP e Realização Pessoal - RP). Métodos: Estudo transversal, observacional; aplicados questionários de novembro a dezembro de 2015 constituídos pelo Maslach Burnout Inventory e por variáveis sociodemográficas e da residência. Tamanho da amostra estimado de 327 residentes (IC 95\%; erro amostral de $5 \%$ ). Resultados: Amostra representativa composta por 431 residentes (média de 28,7 anos, $80,7 \%$ do gênero feminino). A prevalência de burnout global (níveis de burnout elevado na dimensão da EE e/ou DP) foi $46,9 \% ; 38,1 \%$ dos residentes apresentava burnout elevado na EE, 45,2\% na RP e $26,5 \%$ na DP. Verificou-se uma associação estatisticamente significativa entre níveis de burnout elevado e residentes medicados com ansiolíticos/hipnóticos EE $(p<0,001)$; RP $(p=0,001)$ e DP $(p<0,001)$ e também nos residentes medicados com antidepressivos, na EE e $\operatorname{RP}(p=0,01)$. Nos residentes com intenção de desistir da residência/carreira médica verificaram-se níveis elevados de burnout na $\operatorname{EE}(p<0,001$ para ambas), na RP $(p=0,003$ e $p=0,01$, respectivamente) e na DP ( $p=0,005$ e $p<0,001$, respectivamente). Nos residentes que não escolheram MGF como primeira opção, verificaram-se níveis de burnout elevados na dimensão da DP $(p<0,001)$ e da RP $(p=0,04)$. Conclusão: Dada a elevada prevalência de burnout nos residentes de MGF em Portugal, torna-se fundamental desenvolver novos estudos a nível internacional e desenvolver estratégias que previnam e minimizem o impacto negativo desta síndrome.

\section{Abstract}

Objective: To determine the burnout prevalence among general and family medicine residents (MGF) in Portugal and to analyze variables that can influence burnout levels in their three dimensions (Emotional Exhaustion - EE; Depersonalization - DP; and Personal Accomplishment - PA). Methods: Cross-sectional study observation; questionnaires were apllied from November to December 2015, constituted by the Maslach Burnout Inventory and by sociodemographic and residency variables. Sample size estimated of 327 residents (Cl $95 \%$; sampling error of $5 \%$ ). Results: The representative sample of 431 residents (average age 28.7 years old, $80.7 \%$ comprised of women). The prevalence of overall burnout (high burnout levels in EE and/or DP) was $46.9 \%$, with $38.1 \%$ with high burnout in EE dimension, $45.2 \%$ in PA dimension and $26.5 \%$ in DP dimension. There was a statistically significant association between high burnout levels in residents taking anxiolytics/hypnotics - EE $(p<0.001)$; PA $(p=0.001)$ e DP $(p<0.001)$ and in residents taking antidepressant medication - in EE and PA dimensions $(p=0.01)$. In residents intending to give up the internship or the medical career we found high levels of burnout in $E E$ ( $p<0.001$ for both intentions), in PA ( $p=0.003$ and $p=0.01$, respectively) and DP ( $p=0.005$ and $p<0.001$, respectively). The residents who did not choose family medicine (MGF) as a first option had high levels of burnout in DP $(p<0.001)$ and PA $(p=0.04)$ dimensions. Conclusions: Given the high prevalence of burnout in family medicine residents in Portugal, it is essential to develop new studies internationally and to create strategies to prevent and minimize the negative impact of this syndrome.

Como citar: Santos SCR, Viegas AIF, Morgado CIMO, Ramos CSV, Soares CND, Roxo HMCJ, et al. Prevalência de burnout em médicos residentes de Medicina Geral e Familiar em Portugal. Rev Bras Med Fam Comunidade. 2017;12(39):1-9. http://dx.doi.org/10.5712/rbmfc12(39)1430
Palavras-chave: Esgotamento Profissional Internato e Residência Medicina de Família e Comunidade Atenção Primária à Saúde

Keywords: Burnout, Professional Internship and Residency Family Practice Primary Health Care

Fonte de financiamento: declaram não haver. Parecer CEP: Proc.071/CES/INV/2015 (ARSLVT), aprovado em 06/11/2015.

Conflito de interesses: declaram não haver. Procedência e revisão por pares: revisado por pares. Recebido em: 26/11/2016. Aprovado em: 12/03/2017. 


\section{Resumen}

Objetivos: Determinar la prevalencia del síndrome de burnout en médicos residentes de Medicina Familiar (MF) en Portugal y analizar variables que pudieran influir en sus tres dimensiones (Agotamiento Emocional - CE; Despersonalización - DP; Realización Personal - RP). Métodos: Estudio transversal, observacional; cuestionarios aplicados de noviembre a diciembre de 2015, constituidos por el Maslach Burnout Inventory y variables sociodemográficas y de la residencia. Tamaño de la muestra estimada de 327 residentes (IC 95\%; error muestral de $5 \%$ ). Resultados: Muestra representativa de 431 residentes (edad promedio 28,7 años, $80,7 \%$ sexo femenino). La prevalencia de burnout global (altos niveles de burnout en CE y/o DP) fue del 46,9\%; tenían niveles altos de burnout en CE $(38,1 \%)$, RP $(45,2 \%)$ y DP $(26,5 \%)$. Se observó una asociación estadísticamente significativa entre niveles elevados de burnout y residentes tratados con ansiolíticos/hipnóticos - $C E(p<0,001)$; $R P(p=0,001)$, DP $(p<0,001)$ y en los residentes tratados con antidepresivos en CE y RP $(p=0,01)$. En los que querían renunciar a la especialidad/ carrera médica, encontramos altos niveles de burnout en $C E(p<0,001), \operatorname{RP}(p=0,003$ y $p=0,01$, respectivamente) y DP ( $p=0,005$ y $p<0,001$, respectivamente). En los residentes que no eligieron MF como primera opción, encontramos altos niveles de burnout en DP $(p<0,001)$ y $\operatorname{RP}(p=0,04)$. Conclusión: Dada la alta prevalencia del burnout en los residentes de MF en Portugal, es esencial desarrollar nuevos estudios a nivel internacional y desarrollar estrategias para la prevención del impacto negativo de este síndrome.
Palabras clave:

Agotamiento Profesional

Internado y Residencia

Medicina Familiar

y Comunitaria

Atención Primaria de Salud

\section{Introdução}

Nos últimos anos têm sido realizados diversos estudos a nível mundial sobre a síndrome de desgaste profissional, também conhecida como burnout. ${ }^{1-4}$ Esta caracteriza-se por uma tríade de Exaustão Emocional (EE) - perda de capacidade e/ou de recursos emocionais para lidar com o outro; Despersonalização (DP) resposta negativa, de cinismo e distanciamento em relação aos usuários e colegas de trabalho; baixa Realização Pessoal (RP) - insatisfação e percepção de falta de competência no desempenho laboral. ${ }^{1-4}$ Estas três dimensões podem ser avaliadas com o uso do questionário Maslach Burnout Inventory-Human Services Survey (MBI-HSS), que tem sido considerado como o padrão-ouro no diagnóstico de burnout. ${ }^{5}$

A síndrome de burnout é multifatorial e tem elevada prevalência nos médicos. Um estudo europeu realizado em larga escala - European General Practice Research Network Burnout Study - verificou que $43 \%$ dos médicos apresentaram pontuações elevadas de burnout na EE, 35\% na DP e 32\% na RP; $12 \%$ apresentaram pontuações elevadas em todas as dimensões.

Vários fatores parecem estar associados a níveis mais elevados de burnout, tais como a especialidade médica (sendo maior nos médicos de família, de medicina interna e medicina de urgência), o país de trabalho, o abuso do álcool, tabaco e medicação psicotrópica, o gênero e a idade. Além disso, os médicos residentes apresentam níveis de burnout superiores aos dos especialistas, sendo maior também no início da carreira. ${ }^{6-14}$

A maior vulnerabilidade dos médicos residentes ao burnout pode explicar-se pela falta de experiência, pelos constrangimentos relacionados com a organização da residência, pelo elevado grau de responsabilidade associado a um baixo grau de autonomia e pelo elevado grau de dependência das avaliações para concluírem a sua formação. ${ }^{6-13}$

O burnout tem um reconhecido impacto negativo, quer na saúde dos médicos residentes, quer na prática clínica e na relação médico-usuário: está associado à diminuição da satisfação do médico e do usuário, à perda de empatia, à diminuição da produtividade, ao aumento da taxa de absentismo laboral, à desistência da carreira médica e a erros médicos. Além disso, pode condicionar repercussões sociofamiliares, abuso de substâncias, depressão, ou mesmo ideação suicida. ${ }^{9-15}$ 
Dada a elevada prevalência e o impacto do burnout, o objetivo deste trabalho de investigação foi determinar a prevalência de burnout nos residentes de Medicina Geral e Familiar (MGF) em Portugal. Pretendeu-se, também, analisar algumas variáveis sociodemográficas que possam influenciar positiva ou negativamente a pontuação nas três dimensões avaliadas pelo MBI-HSS. Em última análise, determinar a prevalência do burnout permitirá compreender melhor que fatores lhe estão associados e propor estratégias e intervenções que minimizem o seu impacto negativo e, consequentemente, melhorem a qualidade dos cuidados de saúde que prestamos aos nossos usuários. ${ }^{15-19}$

\section{Métodos}

Elaborou-se um estudo transversal e observacional. A amostra foi constituída por médicos residentes de MGF em Portugal que aceitaram preencher o questionário aplicado no período de novembro a dezembro de 2015. O tamanho da amostra foi calculado com base no outcome primário - determinação da prevalência de burnout nos residentes de MGF em Portugal - com recurso às listas dos residentes colocados por especialidade de 2010 a 2014 a nível nacional, perfazendo um total de 2175 residentes.

Considerando um intervalo de confiança de 95\% e um erro amostral de 5\%, para a amostra ser estatisticamente representativa, estimou-se ser necessário obter um total de 327 respostas. Os critérios de inclusão estabelecidos foram: ser residente de MGF em Portugal e a vontade em participar no estudo.

Foi aplicado um questionário anônimo e confidencial, por meio do instrumento Google Forms, constituído pela versão portuguesa e validada do MBI-HSS e um questionário que avaliou as seguintes variáveis: idade (em anos), gênero, estado civil, número de filhos, ano de residência, local de formação (por Agrupamentos de Centros de Saúde e Administração Regional de Saúde), escolha da especialidade de MGF como primeira opção, intenção de desistir do residência, intenção de desistir da carreira médica, uso atual de antidepressivos e uso atual de ansiolíticos/hipnóticos.

O MBI-HSS avalia as três dimensões do burnout de acordo com uma escala de Likert, com nove perguntas relativas à $E E$, cinco à $\mathrm{DP}$ e oito à RP. A pontuação foi obtida para cada dimensão de forma independente classificada como nível baixo, intermédio ou elevado. Também calculou-se o burnout global (overall burnout), que foi considerado como níveis de burnout elevados na dimensão da EE e/ou da DP.

Ao contrário das dimensões da EE e da DP, no caso da RP quanto mais baixa a pontuação, mais elevados são os níveis de burnout.

Para análise dos dados obtidos, estes foram inicialmente organizados numa base de dados do Microsoft Excel 2013 e posteriormente a análise estatística foi realizada com o uso do programa Statistical Package for the Social Sciences (SPSS ${ }^{\circledR}$ ), versão 20.0 para Microsoft Windows. Todas as variáveis sociodemográficas, caracterização da residência médica e hábitos medicamentosos foram sumarizados por meio de estatísticas descritivas: frequências absolutas e percentagens para variáveis qualitativas e média, desvio padrão, mediana, mínimo e máximo para variáveis quantitativas.

A prevalência de burnout foi estimada para o total da amostra e por gênero, faixa etária, estado civil, número de filhos, características da residência médica e hábitos medicamentosos, com intervalos de confiança a 95\%, por meio de análises bivariadas usando o teste do Qui-Quadrado. 
O protocolo do presente estudo foi submetido à Comissão de Ética da Administração Regional de Saúde de Lisboa e Vale do Tejo, cujo parecer foi favorável à realização do estudo.

\section{Resultados}

De acordo com o cálculo da amostra, obteve-se um total de 431 respostas, todas válidas. A maioria dos inquiridos tinham idades compreendidas entre 25 e 29 anos (76,8\%), com média de 28,7 anos, sendo a maioria do gênero feminino $(80,7 \%)$ - Quadro 1 .

Quadro 1. Caraterização da amostra sociodemográfica e da residência médica ( $n=431)$.

\begin{tabular}{|c|c|c|c|c|c|c|c|c|c|c|c|c|}
\hline \multirow[t]{2}{*}{ Variável } & \multicolumn{3}{|c|}{ Faixa etária (anos) } & \multicolumn{2}{|c|}{ Gênero } & \multicolumn{4}{|c|}{ Estado civil } & \multicolumn{3}{|c|}{ Número de filhos } \\
\hline & $25-29$ & $30-34$ & $\geq 35$ & Masculino & Feminino & Solteiro(a) & $\begin{array}{l}\text { Casado(a)/ } \\
\text { União de facto }\end{array}$ & Divorciado(a) & Viúvo(a) & 0 & 1 & 2 \\
\hline $\mathrm{N}$ & 331 & 91 & 9 & 83 & 348 & 268 & 160 & 2 & 1 & 383 & 39 & 9 \\
\hline$\%$ & 76,8 & 21,1 & 2,1 & 19,3 & 80,7 & 62,2 & 37,1 & 0,5 & 0,2 & 88,9 & 9,0 & 2,1 \\
\hline \multirow[t]{2}{*}{ Variável } & \multicolumn{5}{|c|}{ Ano de internato } & \multicolumn{2}{|c|}{ MGF como $1^{\text {a }}$ opção } & \multicolumn{2}{|c|}{$\begin{array}{l}\text { Intenção de desistir } \\
\text { da residência }\end{array}$} & \multicolumn{3}{|c|}{$\begin{array}{l}\text { Intenção de desistir } \\
\text { da carreira médica }\end{array}$} \\
\hline & \multicolumn{2}{|c|}{10} & $2^{\circ}$ & $3^{\circ}$ & $4^{\circ}$ & Sim & Não & Sim & Não & Sim & \multicolumn{2}{|c|}{ Não } \\
\hline $\mathrm{N}$ & \multicolumn{2}{|c|}{100} & 118 & 102 & 111 & 362 & 69 & 26 & 405 & 24 & \multicolumn{2}{|c|}{407} \\
\hline$\%$ & \multicolumn{2}{|c|}{23,2} & 27,4 & 23,7 & 25,8 & 84 & 16 & 6 & 94 & 5,6 & \multicolumn{2}{|c|}{94,4} \\
\hline
\end{tabular}

Verificou-se uma distribuição equitativa por anos de residência e obtiveram-se respostas de residentes de todas as Administrações Regionais de Saúde (ARS).

A MGF foi a primeira opção de escolha de especialidade para $84,0 \%$ dos residentes. Verificou-se que $6,0 \%$ tinham intenção de desistir da residência e 5,6\% da carreira médica.

O uso de medicamentos hipnóticos durante o residência foi reportado por $11,1 \%$ da amostra e o uso de antidepressivos por $10,2 \%$.

A prevalência de burnout global (overall burnout), considerado como burnout elevado nas dimensões de EE e/ou DP, foi de 46,9\%. Verificou-se que 38,1\% dos residentes apresentavam níveis de burnout elevado na dimensão EE, 45,2\% na dimensão da RP e $26,5 \%$ na dimensão de DP (Figura 1).

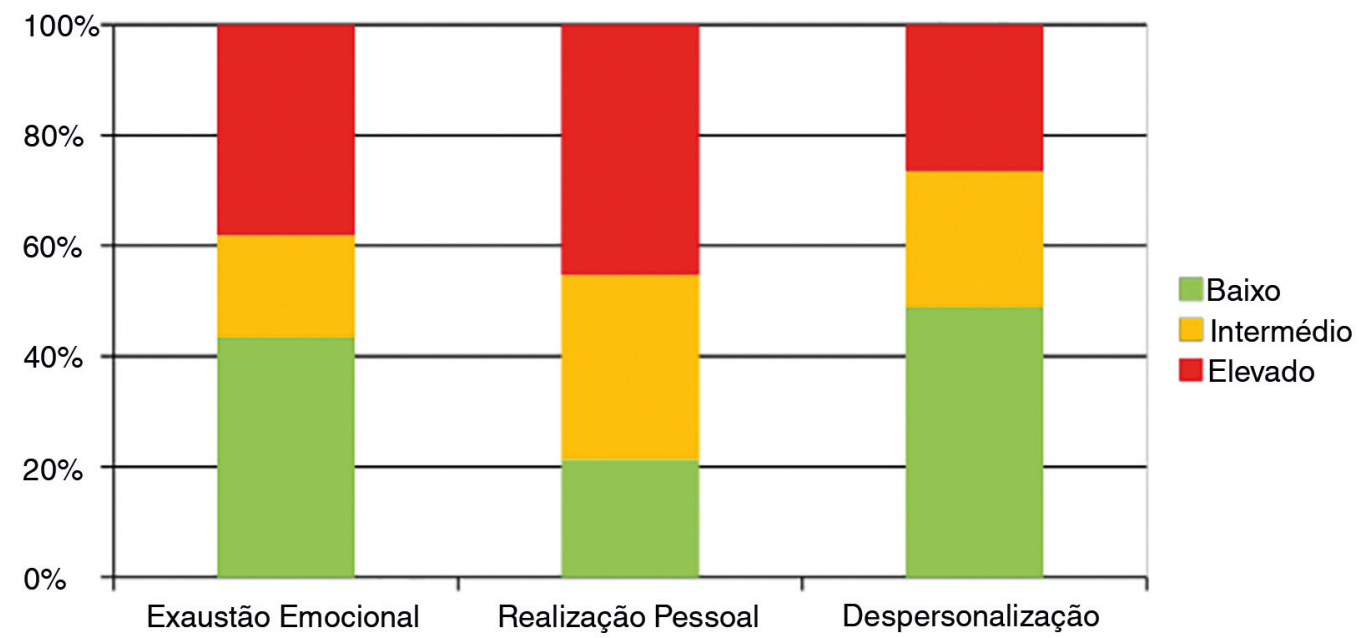

Figura 1. Prevalência de burnout por dimensão nos residentes de Medicina Geral e Familiar em Portugal. 
Verificou-se que $63,8 \%$ dos médicos residentes inquiridos apresentavam níveis de burnout elevados em pelo menos uma das dimensões avaliadas, $11,8 \%$ apresentavam níveis elevados nas três dimensões e $36,2 \%$ não obtiveram níveis elevados de burnout em qualquer dimensão.

$\mathrm{Na}$ análise comparativa entre os níveis de burnout e as características da residência encontramos associações estatisticamente significativas. A intenção de desistir da residência e a intenção de desistir da carreira médica estão associadas a níveis de burnout elevados, quer considerando o burnout global ( $p=0,002$ e $p<0,001$ ), quer considerando cada dimensão de forma independente (Quadro 2). Os residentes que não escolheram MGF como primeira opção para especialidade médica apresentaram níveis de burnout mais elevados na dimensão da $\operatorname{RP}(p=0,04)$ e da $\operatorname{DP}(p<0,001)$, quando comparados àqueles para os quais a MGF foi a primeira escolha de carreira médica.

Quadro 2. Análise comparativa dos níveis de burnout por dimensão e de burnout global nas variáveis estatisticamente significativas.

\begin{tabular}{|c|c|c|c|c|c|c|c|c|c|}
\hline \multirow[t]{2}{*}{$\begin{array}{l}\text { Parãmetro de } \\
\text { burnout }\end{array}$} & \multirow[t]{2}{*}{$\begin{array}{l}\text { Nível de } \\
\text { burnout }\end{array}$} & \multicolumn{2}{|c|}{$\begin{array}{l}\text { Intenção de desistir } \\
\text { da residência }\end{array}$} & \multicolumn{2}{|c|}{$\begin{array}{l}\text { Intenção de desistir } \\
\text { da carreira médica }\end{array}$} & \multicolumn{2}{|c|}{$\begin{array}{c}\text { Toma de } \\
\text { ansiolítico/hipnótico }\end{array}$} & \multicolumn{2}{|c|}{$\begin{array}{c}\text { Toma de } \\
\text { antidepressivo }\end{array}$} \\
\hline & & $\operatorname{Sim}(\%)$ & Não (\%) & $\operatorname{Sim}(\%)$ & Não (\%) & $\operatorname{Sim}(\%)$ & Não (\%) & $\operatorname{Sim}(\%)$ & Não (\%) \\
\hline \multirow[t]{2}{*}{ Exaustão emocional } & Intermédio & 7,7 & 19,3 & 8,3 & 19,2 & 6,3 & 20,1 & 15,9 & 18,9 \\
\hline & Elevado & 73,1 & 35,8 & 79,2 & 35,6 & 79,2 & 32,9 & 52,3 & 36,4 \\
\hline Valor $p$ & Baixo & 0 & 22,7 & 4,2 & 22,4 & 14,6 & 22,2 & 6,8 & 23,0 \\
\hline \multirow[t]{2}{*}{ Realização pessoal } & Intermédio & 26,9 & 33,8 & 25,0 & 33,9 & 16,7 & 35,5 & 29,5 & 33,9 \\
\hline & Elevado & 73,1 & 43,5 & 43,7 & 43,7 & 68,8 & 42,3 & 63,6 & 43,2 \\
\hline \multicolumn{2}{|l|}{ Valor $\mathrm{p}$} & \multicolumn{2}{|c|}{0,003} & \multicolumn{2}{|c|}{0,010} & \multicolumn{2}{|c|}{0,001} & \multicolumn{2}{|c|}{0,010} \\
\hline Despersonalização & Baixo & 34,6 & 49,9 & 16,7 & 50,9 & 29,2 & 51,4 & 47,7 & 49,1 \\
\hline \multirow{2}{*}{ Burnout global } & Sim & 76,9 & 44,9 & 87,5 & 44,5 & 83,3 & 42,3 & 63,6 & 45,0 \\
\hline & Não & 23,1 & 55,1 & 12,5 & 55,5 & 16,7 & 57,7 & 36,4 & 55,0 \\
\hline Valor $p$ & & \multicolumn{2}{|c|}{0,002} & \multicolumn{2}{|c|}{$<0,001$} & \multicolumn{2}{|c|}{$<0,001$} & \multicolumn{2}{|c|}{0,019} \\
\hline
\end{tabular}

Relativamente aos hábitos medicamentosos, verificou-se que o uso de ansiolíticos hipnóticos estava associado a níveis elevados de burnout nas suas três dimensões: $E E(p<0,001) ; \operatorname{RP}(p=0,001)$ e DP $(p<0,001)$ e estava, também, associado a burnout global $(p<0,001)$. Quanto ao uso de medicação antidepressiva, constatou-se uma associação com níveis elevados de burnout nas dimensões de EE e RP $(p=0,01)$ e com o burnout global $(p=0,019)$.

Relativamente à análise comparativa entre os níveis de burnout e as características sociodemográficas da amostra, não foi encontrada nenhuma associação estatisticamente significativa, quer considerando cada dimensão de burnout individualmente, quer considerando o burnout global.

Relativamente ao ano de residência, não foi observada associação estatisticamente significativa com o nível de burnout global elevado $(p=0,591)$, contudo, constatou-se uma prevalência crescente nos níveis de burnout global com a progressão no ano de residência. 


\section{Discussão}

De acordo com diversos estudos internacionais, a prevalência de burnout em residentes é variável $(18-82 \%),{ }^{10}$ sendo utilizadas diferentes escalas de avaliação, com diferentes interpretações, em particular na definição do burnout global. A maioria dos estudos utiliza o MBI-HSS, ${ }^{10}$ tendo sido também essa a nossa opção.

No nosso estudo, a prevalência de burnout global foi de $46,9 \%$, sendo importante salientar que $63,8 \%$ dos médicos residentes apresentavam níveis de burnout elevados em pelo menos uma das dimensões avaliadas. Este resultado está de acordo com outros estudos internacionais, em que mais de $50 \%$ dos residentes apresentou níveis elevados em pelo menos uma dimensão. ${ }^{10}$ Contudo, se considerarmos uma definição mais restrita de burnout global, em que seria necessário apresentar níveis elevados em todas as dimensões do MBI-HSS, a taxa de burnout na nossa amostra desceria para os $11,8 \%$, sendo, ainda assim, superior às descritas em estudos internacionais. ${ }^{11}$

A pertinência do tema é clara, em especial pelas suas potenciais repercussões negativas a nível pessoal e profissional do burnout, como o maior risco de erro médico e a prestação de cuidados de saúde subótimos aos usuários. ${ }^{6,12,13}$ Assim, a prevenção do burnout é importante não apenas para os profissionais de saúde, mas também para os usuários.

Existe um estudo sobre Burnout em Profissionais de Saúde Portugueses, ${ }^{19} \mathrm{e}$, até a presente data, foi publicado a nível nacional apenas um estudo sobre residentes de MGF, realizado em 2009 e com uma amostra de conveniência de 210 residentes da região norte de Portugal. ${ }^{20}$

Embora não possam ser realizadas comparações estatísticas, salientamos que o número de residentes analisados nesta região foi semelhante (o nosso estudo incluiu 217 residentes da Administração Regional de Saúde do Norte versus 210 residentes no estudo acima referido), mas a prevalência de burnout global encontrada no nosso estudo correspondente a esta região Norte $(50,2 \%)$ foi superior à do estudo realizado na zona Norte de Portugal em 2009 (34,3\%).

Estes dados reforçam a pertinência do nosso trabalho, realizado em 2015, exclusivamente com residentes de MGF, abrangendo todos os anos de residência, nos diversos modelos organizacionais de CSP portugueses (UCSP e USF) e que incluiu as várias regiões de Portugal continental e regiões autônomas.

Neste estudo foi encontrada uma associação estatisticamente significativa entre o burnout e o uso de medicamentos ansiolíticos hipnóticos e antidepressivos, e com a intenção de desistir da residência ou da carreira médica. Estes dados traduzem o impacto negativo do burnout no estado de saúde dos profissionais, também descrito em outros estudos. ${ }^{6}$

Diversos estudos constatam que a síndrome de burnout é muito prevalente nos profissionais de saúde, sendo mais frequente nas fases mais precoces da carreira. ${ }^{6,13,18}$ Estudos recentes sugerem níveis mais elevados de burnout em especialidades na primeira linha de acesso aos cuidados médicos, em que se inclui a $M G F,{ }^{13}$ não se sabendo se esses dados podem ser extrapolados à residência médica. Sabe-se, contudo, que quando presente no início da residência, a síndrome de burnout tende a persistir ao longo da carreira médica. ${ }^{10} \mathrm{O}$ interesse na quantificação do burnout nos residentes tem aumentado, não sendo possível extrapolar os resultados, uma vez que os níveis de burnout variam consoante as especificidades culturais, educacionais e organizacionais de cada país. ${ }^{13,17}$ 
Os níveis elevados de burnout na residência de MGF poderão estar relacionados com características próprias à residência desta especialidade, nomeadamente, a abrangência da especialidade e a frequente incerteza diagnóstica, a exigência emocional da relação médico-usuário-família e a aquisição precoce de autonomia. Neste sentido, torna-se fundamental implementar medidas que promovam o bem-estar desde uma fase precoce da residência médica.

No nosso estudo não foi encontrada nenhuma associação estatisticamente significativa entre o nível de burnout global elevado e as características sociodemográficas da amostra ou o ano de residência, embora se tenha constatado uma prevalência crescente nos níveis de burnout global com a progressão no ano de residência.

Estes resultados podem ser explicados pelos efeitos de um aumento na carga de trabalho, menor supervisão com maior autonomia, a dependência de uma avaliação contínua, maior exigência emocional e dificuldade na gestão da vida profissional e pessoal. ${ }^{10}$ Poderão também relacionar-se com a realização de um esforço adicional para alcançar melhores resultados para a colocação na vaga como médico especialista no local de trabalho pretendido, o que pode aumentar a competitividade. ${ }^{13}$

Os níveis de burnout elevados encontrados nas dimensões de RP $(56,5 \%)$ e DP $(43,5 \%)$ em residentes que não escolheram MGF como primeira opção sugerem que colegas que preferiam outra especialidade podem não se sentir realizados profissionalmente, podendo estar mais vulneráveis a desenvolver sentimentos de cinismo e de distanciamento/frieza face aos usuários e aos colegas de trabalho, evidenciando a importância da vocação na escolha da especialidade médica.

Ainda assim, a MGF foi primeira opção de escolha de especialidade para $84 \%$ dos inquiridos e a prevalência de burnout global foi de 46,9\%. Poderá existir uma percentagem de residentes cuja escolha terá sido baseada na imagem da especialidade criada durante o período de Faculdade, que pode não corresponder posteriormente às expectativas criadas. Assim, como potencial medida de prevenção de burnout, seria pertinente a inclusão e revisão dos programas curriculares desta especialidade nas faculdades de Medicina. ${ }^{21}$

As características do orientador de formação podem condicionar a motivação profissional dos residentes ao longo do seu percurso na residência, estando descrito que os mesmos valorizam o apoio que lhes é fornecido durante este período. ${ }^{22}$ Assim, a seleção cuidadosa de orientadores e atenção na formação educacional dos residentes poderão ser estratégias a considerar na prevenção de burnout.

Como limitações deste estudo, salientamos um potencial viés na seleção da amostra por residentes em burnout, que podem ter estado menos motivados para o preenchimento do questionário ou, por outro lado, terem estado mais interessados no tema e serem mais participativos.

Além disso, é importante considerar um possível viés de aferição, apesar da utilização de um instrumento validado para a população portuguesa (MBI-HSS).

Outra limitação reside no fato de o estudo ser transversal. Embora este tipo de estudo seja útil a nível populacional, serão necessários estudos longitudinais para aferir com maior precisão os efeitos do burnout nas suas várias dimensões ao longo da residência médica.

À semelhança de outros estudos, poderiam ter sido avaliadas outras variáveis que não foram incluídas, como dependências (consumo de álcool ou drogas), depressão e ideação suicida, número de horas de trabalho extra (privado, serviço de urgência), número de trabalhos realizados na residência, reconhecimento por parte do orientador, da equipe de saúde e dos usuários, tempo dedicado à família e amigos considerado suficiente e atividades de lazer, entre outras. 
O tipo de estudo realizado não permitiu identificar as causas de burnout. O conhecimento das causas de burnout poderá permitir estabelecer estratégias de coping mais eficazes. ${ }^{6,12}$

Neste estudo foi apenas determinada a prevalência de burnout em residentes de MGF e dada a sua pertinência será de todo o interesse a realização de um estudo mais abrangente de médicos residentes e especialistas dos cuidados de saúde primários e secundários, quer a nível nacional, quer a nível internacional.

Os resultados obtidos neste estudo são preocupantes, uma vez que o burnout pode dificultar os processos cognitivos necessários para a aquisição de conhecimentos e técnicas, ${ }^{13}$ podendo mesmo comprometer a aprendizagem que se pressupõe inerente ao período de residência, com riscos para a prática clínica e, consequentemente, para os usuários. Estes resultados alertam para a necessidade urgente de criação de estratégias precoces de prevenção do burnout, nas faculdades e no programa de residência médica desta especialidade.

Existe evidência de que intervenções orientadas para a organização do trabalho ou para uma abordagem individual junto dos médicos como, por exemplo, técnicas de mindfulness ou gestão de stress podem ser eficazes para reduzir o burnout nos médicos. ${ }^{23}$ Seria interessante implementar estas técnicas em Unidades de Saúde e perceber o impacto das mesmas na redução do burnout nos residentes de MGF.

Serão necessários mais estudos para definir as causas de burnout nos residentes de MGF em Portugal e quais os tipos de intervenção mais adequados a implementar em prol da saúde e do bem-estar dos usuários e dos futuros médicos de família portugueses. . $^{6,19}$

\section{Agradecimentos}

Aos residentes de MGF de todo o país, que se disponibilizaram a responder ao questionário.

Agradecemos também à CLINICAL LAB, em particular a Dra. Catarina Silva, pela contribuição na análise estatística.

\section{Referências}

1. Soler JK, Yaman H, Esteva M, Dobbs F, Asenova RS, Katic M, et al.; European General Practice Research Network Burnout Study Group. Burnout in European family doctors: the EGPRN study. Fam Pract. 2008;25(4):245-65. DOI: http://dx.doi. org/10.1093/fampra/cmn038

2. Morelli SG, Sapede M, Silva ATC. Burnout em médicos da Atenção Primária: uma revisão sistemática. Rev Bras Med Fam Comunidade. 2015;10(34):1-9. DOI: http://dx.doi.org/10.5712/rbmfc10(34)958

3. Putnik K, Houkes I. Work related characteristics, work-home and home-work interference and burnout among primary healthcare physicians: a gender perspective in a Serbian context. BMC Public Health. 2011;11:716. DOI: http://dx.doi. org/10.1186/1471-2458-11-716

4. Matía Cubillo AC, Cordero Guevara J, Mediavilla Bravo JJ, Pereda Riguera MJ, González Castro ML, González Sanz A. Evolution of burnout and associated factors in primary care physicians. Aten Primaria. 2012;44(9):532-9.

5. Al-Sareai NS, Al-Khaldi YM, Mostafa OA, Abdel-Fattah MM. Magnitude and risk factors for burnout among primary health care physicians in Asir Province, Saudi Arabia. East Mediterr Health J. 2013;19(5):426-34.

6. Schaufeli WB, Bakker AB, Hoogduin K, Schaap C, Kladler A. on the clinical validity of the maslach burnout inventory and the burnout measure. Psychol Health. 2001;16(5):565-82. DOI: http://dx.doi.org/10.1080/08870440108405527

7. Prins JT, Gazendam-Donofrio SM, Tubben BJ, van der Heijden FM, van de Wiel HB, Hoekstra-Weebers JE. Burnout in medical residents: a review. Med Educ. 2007;41(8):788-800. DOI: http://dx.doi.org/10.1111/j.1365-2923.2007.02797.x 
8. Ishak WW, Lederer S, Mandili C, Nikravesh R, Seligman L, Vasa M, et al. Burnout during residency training: a literature review. J Grad Med Educ. 2009;1(2):236-42. DOI: http://dx.doi.org/10.4300/JGME-D-09-00054.1

9. Thomas NT. Resident burnout. JAMA. 2004;292(23):2880-9. DOI: http://dx.doi.org/10.1001/jama.292.23.2880

10. Galam E, Komly V, Le Tourneur A, Jund J. Burnout among French GPs in training: a cross-sectional study. Br J Gen Pract. 2013;63(608):e217-24. DOI: http://dx.doi.org/10.3399/bjgp13X664270

11. Zis P, Anagnostopoulos F, Sykioti P. Burnout in medical residents: a study based on the job demands-resources model. ScientificWorldJournal. 2014;2014:673279. DOI: http://dx. doi.org/10.1155/2014/673279

12. Dyrbye LN, West CP, Satele D, Boone S, Tan L, Sloan J, et al. Burnout among U.S. medical students, residents, and early career physicians relative to the general U.S. population. Acad Med. 2014;89(3):443-51. DOI: http://dx.doi.org/10.1097/ ACM.0000000000000134

13. Rutherford K, Oda J. Family medicine residency training and burnout: a qualitative study. Can Med Educ J.2014;5(1):e13-23.

14. Dyrbye L, Shanafelt T. A narrative review on burnout experienced by medical students and residents. Med Educ. 2016;50(1):132-49. DOI: http://dx. doi.org/10.1111/medu.12927

15. Prins JT, Hoekstra-Weebers JE, van de Wiel HB, Gazendam-Donofrio SM, Sprangers F, Jaspers FC, et al. Burnout among Dutch medical residents. Int J Behav Med. 2007;14(3):119-25. DOI: http://dx.doi.org/10.1007/BF03000182

16. Gómez-Gascón T, Martín-Fernández J, Gálvez-Herrer M, Tapias-Merino E, Beamud-Lagos M, Mingote-Adán JC; Grupo EDESPROAP-Madrid. Effectiveness of an intervention for prevention and treatment of burnout in primary health care professionals. BMC Fam Pract. 2013;14:173. DOI: http://dx.doi.org/10.1186/1471-2296-14-173

17. Bovier PA, Arigoni F, Schneider M, Gallacchi MB. Relationships between work satisfaction, emotional exhaustion and mental health among Swiss primary care physicians. Eur J Public Health. 2009;19(6):611-7. DOI: http://dx.doi.org/10.1093/ eurpub/ckp056

18. Nielsen HG, Tulinius C. Preventing burnout among general practitioners: is there a possible route? Educ Prim Care. 2009;20(5):353-9.

19. Marôco J, Marôco AL, Leite E, Bastos C, Vazão MJ, Campos J. Burnout em Profissionais de Saúde Portugueses: Uma Análise a Nível Nacional. Acta Med Port. 2016;29(1):24-30. DOI: http://dx.doi.org/10.20344/amp.6460

20. Mendes P, Cardoso VP, Yaphe J. Stress e burnout em internos de medicina geral e familiar da zona Norte de Portugal: estudo transversal. Rev Port Med Geral Fam. 2017;33(1):16-28.

21. Gaspar D. Medicina Geral e Familiar: uma escolha gratificante. Acta Med Port. 2006;19(2):133-40.

22. Gaspar D, Jesus SN, Cruz JP. Motivação Profissional e Apoio Fornecido no Estágio: Estudo longitudinal de uma Coorte de Médicos do Internato Médico de Medicina Geral e Familiar. Acta Med Port. 2011;24(S2):137-46.

23. West CP, Dyrbye LN, Erwin PJ, Shanafelt TD. Interventions to prevent and reduce physician burnout: a systematic review and meta-analysis. Lancet. 2016;388(10057):2272-81. DOI: http://dx.doi.org/10.1016/S0140-6736(16)31279-X

a Unidade de Saúde Familiar S. Julião (Agrupamento de Centros de Saúde Lisboa Ocidental e Oeiras). Portugal. sc.robalosantos@gmail.com (Autora correspondente); catarinaimoliveira@gmail.com; carlasof.ramos@gmail.com; mafcss@gmail.com

b Unidade de Saúde Familiar Conde de Oeiras (Agrupamento de Centros de Saúde Lisboa Ocidental e Oeiras).Portugal. anaifviegas@gmail.com; sarannabais@gmail.com

c Unidade de Saúde Familiar Travessa da Saúde (Agrupamento de Centros de Saúde Loures Odivelas). Portugal. christinalee804@gmail.com

d Unidade de Saúde Familiar Cova Piedade (Agrupamento de Centros de Saúde Almada Seixal). Portugal. mafaldaroxo@live.com 